\title{
Chest computed tomography: is it ready for major studies of chronic obstructive pulmonary disease?
}

\author{
M.G. Cosio*, G.L. Snider ${ }^{\#}$
}

Chronic obstructive pulmonary disease (COPD) requires for diagnosis the presence of chronic airflow limitation and emphysema or chronic bronchitis. In the USA, the disease causes $>100,000$ deaths annually, it is the fourth most important cause of death and afflicts about 16 million persons. About 1\% of COPD in the USA is associated with $\alpha_{1}$-antitrypsin $\left(\alpha_{1}-\mathrm{AT}\right)$ deficiency and is conveniently referred to as $\alpha_{1}$-AT-COPD. This form of the disease occurs in younger persons, progresses more rapidly, is associated with a higher frequency of panlobular emphysema (PLE) than usual COPD, and may be treated by augmentation therapy with concentrated human $\alpha_{1}$-AT. However, there is limited evidence that $\alpha_{1}$-AT replacement modifies the rate of progression of $\alpha_{1}$-AT-COPD.

How can the rate of progression be monitored? Using tools derived from usual COPD to monitor $\alpha_{1}$ AT-COPD might not be correct. These diseases share pathophysiological similarities but there are also important differences. $\alpha_{1}$-AT-COPD is mostly due to the rapid development of PLE predominantly in the lower lobes; airflow obstruction and hyperinflation are mainly due to emphysema and losses of elastic recoil. In usual COPD, airflow limitation is largely secondary to airway damage and remodelling and emphysema (mainly centrilobular emphysema (CLE)) predominates in the upper lobes with a heterogeneous distribution in the lung. The extent of CLE is quite variable for a given level of airflow limitation. Moreover, SANDERs et al. [1] and SANTIS et al. [2] found that $68-80 \%$ of smokers showed emphysema by high-resolution computed tomography (HRCT) in the presence of normal lung function tests. These facts highlight the complexity of COPD and the relative independence and at the same time interdependence of airway remodelling and emphysema in their contribution to the development of COPD.

Which end-points should then be used to assess available and future new drugs for the treatment of both forms of COPD? The rate of decline of forced expiratory volume in one second (FEV1), the traditional end-point used, requires a large number of subjects studied over a period of years [3], and does

*McGill University, Royal Victoria Hospital, Montreal, Quebec, Canada. "Boston University School of Medicine, Boston, USA.

Correspondence: M.G. Cosio, McGill University, Royal Victoria Hospital, Respiratory Division, 687 Pine Avenue, West Montreal, Quebec, Canada. Fax: 15148431695 not track the presence and progression of emphysema well enough. FEV1 progression would be difficult to use in $\alpha_{1}$-AT-COPD since the number of patients that could be studied is much smaller and emphysema is the main pathological abnormality, thus it makes sense to measure emphysema and emphysema progression. The computed tomography (CT) scanner could potentially do it.

In 1984, HAYHURST et al. [4] published an important study that was the base for our actual understanding of how to use CT scans for the diagnosis of emphysema. By comparing anatomical emphysema with CT in the same patients, they showed that the distribution curve of attenuated values was significantly shifted toward the lowest attenuation values in emphysematous lungs. Based on this concept, several groups have defined cut-off points in lung attenuation that could best quantify the extent of emphysema in the lungs. As is usually the case, we have ended up with multiple ways of looking at the same issue. Among these, the work by GEvenOIS and coworkers $[5,6]$ stands out for having carefully compared transversal CT slices and transversal anatomical lung slices showing that both macroscopic [5] and microscopic [6] emphysema are well quantitated by CT in smokers using an attenuation cut-off point of -950 Hounsfield units (HU). They also showed that normal lungs can have up to $7 \%$ of attenuation $<-950 \mathrm{HU}$ and this value can increase with increases in total lung capacity and older age [7].

A key question still remaining is whether evidence from CT showing that a drug has delayed destruction of lung tissue by emphysema is sufficient evidence of drug efficacy to satisfy regulatory requirements. The FEV1 is a well established measure of airflow obstruction, which has been shown convincingly to relate to patients' functional status, prognosis and quality of life. What evidence is there that CT-demonstrated severity of emphysema is related to these clinical parameters?

In this issue of the Journal, Dowson et al. [8] attempt to answer these important questions. In a cross-sectional study of HRCT in 111 patients with $\alpha_{1}$-AT deficiency, they determined the relative area of pixels representing lung tissue with a density $<-910 \mathrm{HU}$ for a single upper zone slice (level of the aortic arch) and a single lower zone slice (level of the inferior pulmonary vein). HRCT was performed in both full inspiration and full expiration. In concert with previous studies, they showed moderately strong, 
highly significant correlations between HRCT scan abnormality and FEV1. There were also moderately strong, statistically significant correlations between HRCT pixel indices and other lung function parameters. Expiratory HRCT gave slightly higher correlations than inspiratory values for most spirometric tests, probably indicating that the more abnormal the small airways, the higher the closing volumes and the higher the attenuation. Of interest is that the correlation with carbon monoxide transfer coefficient $(K \mathrm{CO})$, a physiological parameter reflecting the loss in surface area in emphysema, does not change between inspiratory and expiratory scans.

Many studies of CT in usual COPD have shown a relation between CT indices of severity of emphysema and the FEV1; generally, correlation coefficient (rho) values are in the range of -0.7 indicating that only about half the variability of the population have been accounted for. This is far better than the correlations obtained between anatomical emphysema in lung autopsies and FEV1 where correlation coefficients in the order of $0.4-0.5$ were the rule [9]. Airflow obstruction in $\alpha_{1}$-AT-COPD would be mainly due to emphysema and loss of elastic recoil, and in part to collapse at high lung volumes of small airways due to loss of recoil and loss of tethering from the destructive process. It is reassuring that correlation coefficients between CT emphysema and FEV1 as high as -0.70 can also be found in $\alpha_{1}$-AT-COPD. Since, as noted earlier, the FEV1 relates to prognosis and to functional status, it may be postulated by extrapolation that severity of emphysema relates to prognosis, functional status and quality of life.

The study by Dowson et al. [8] provides the first published demonstration of a relation between CT and health status as assessed by the St. George's Respiratory Questionnaire (SGRQ) and the shortform (SF-36) generic measure of functional status and well-being. CT accounted for $10-25 \%$ of the variability of the population's symptom score, activity score, impacts score and total score in the SGRQ; all coefficients of correlation were highly statistically significant. CT also correlated significantly with SF-36 domains relating to bodily pain or emotional and mental health problems. As the authors point out, the relationship between airflow obstruction and health status was even stronger. It would have been of interest to have used multiple correlations analysis to see if emphysema by itself or because of its close relation to FEV1 accounted for health status scores. Still the correlations they obtained are much stronger than those previously noted in usual COPD.

Can CT be considered an appropriate outcome measure in addition to or even replacing responses of FEV 1 to therapy in $\alpha_{1}$-AT-COPD or usual COPD? To answer this, we have to know whether CT can detect emphysema progression and the time-course of change.

A relatively large group of smokers and nonsmokers from Japan were followed by CT scan over 5 yrs to study progression of emphysema [10]. It should be noted that practically all emphysema in usual COPD in Japan is CLE (upper lobes predominant). Using several density parameters, SoEJIMA et al.
[10] found that: 1) densities fall with age progression in nonsmokers; 2) densities fall similarly over time in smokers and past smokers; and 3) densities fall in the upper lung regions but might increase in the lower lung regions, at least in current smokers over the 5-yr period.

DIRKSEN et al. [11] studied 22 patients with $\alpha_{1}$-ATCOPD and moderate emphysema for $2-4$ yrs with an annual lung CT. They used several parameters for the assessment of lung density in both whole lung and single slices. Not surprisingly and opposite to the SoEJIMA et al. [10] study, slices of lung below the carina showed more pronounced changes over time than slices in the lung apex. They found maximum annual changes of mean \pm SEM, $-2.42 \pm 0.40 \mathrm{HU}$ that were statistically significant. But most importantly, they carefully analysed the possible pitfalls of the use of CT scans for comparison purposes. They warn us of how small changes in lung volumes during scanning can result in variations of $-10 \mathrm{HU}$ (their subjects fell by $-2.42 \mathrm{HU}$ a year). Even more importantly, they explain how a variation in the position of the section scanned in a first and second examination could have a most important impact on results.

In a subsequent study, DiRKSEN et al. [12] carried out a double-blind interventional trial of augmentation therapy with $\alpha_{1}$-AT intravenously in 26 Danish and 30 Dutch exsmokers with PIZZ $\alpha_{1}$-AT deficiency for $>3$ yrs. Daily, self-administered spirometry showed no significant difference in decline of FEV1 between treatment and placebo. The degree of emphysema was quantified yearly by the 15 th percentile point of the lung density histogram derived from CT. The loss of lung-tissue density measured by CT, mean $\pm \mathrm{SEM}$, was $2.6 \pm 0.41 \mathrm{~g} \cdot \mathrm{L} \cdot \mathrm{yr}^{-1}$ for placebo as compared with $1.5 \pm 0.41 \mathrm{~g} \cdot \mathrm{L} \cdot \mathrm{yr}^{-1}$ for $\alpha_{1}$-AT infusion $(p=0.07)$. Power analysis showed that this protective effect would be statistically significant in a similar trial with 130 patients. This was in contrast to calculations based on annual decline of FEV1 showing that 550 patients would be needed to show a $50 \%$ reduction of annual decline.

These data are promising. However, this study is a good example of the urgent need for investigators to come together in standardizing the use of CT scans. The CT scanners used in the foregoing study [12] were different. One scanner took $8 \mathrm{~mm}$ thick slices at functional residual capacity (FRC) at scanning parameters of $125 \mathrm{KVP}, 88 \mathrm{~mA}$. The other scanner used $120 \mathrm{KVP}, 250 \mathrm{~mA}, 10 \mathrm{~mm}$ thick slices at $75 \%$ of the total lung capacity. These relatively simple differences might have accounted, at least in part, for the very large variance observed in their study and thus the lack of clearly positive outcomes in their important trial.

We have learned much from the different approaches used by investigators to diagnose emphysema by CT scan. The groups involved in this research are relatively few and a consensus of how to use the technique should be easy to obtain. The new generation of spiral CT scanners already available, have major advantages: the entire thorax is imaged during one single inspiration; lung volume is routinely measured and correlates closely to plethysmographic 
measurements; quantification of lung disorders can be obtained for the whole lung or for selected slices of any desired thickness. Furthermore, the availability of whole lung information at once will facilitate the very precise analysis of lung surface area, lung volume and lung weight as described by Coxson et al. [13]. From these data, it is possible to calculate lung surface to volume ratio, lung density (the ratio of lung weight to volume) and specific volume (the ratio of lung volume to weight). These parameters can be calculated on a "per voxel" basis. This approach permits precisely relating information obtained from CT to morphometric methods applied to lung specimens. Improved methods of following the progression of emphysema and enhanced understanding of the mechanism of progression of emphysema should result.

An authorative statement by radiologists and pneumologists on the current use of CT to follow progression of emphysema is now needed to advance the field. What are the relative merits of CT sections of $10 \mathrm{~mm}$ as opposed to $1 \mathrm{~mm}$ or thereabouts? What is the best computerized way to interpret CT images? What is the best way of limiting radiation exposure in longitudinal studies? What is the signal to noise ratio, that is, can we obtain the same reading for emphysema a few days apart in the same patient? Can we electronically send data from multiple scanners to a central station for further analysis?

Although the technology of making CT images of the chest continues to evolve at a rapid pace, the evidence suggests that if techniques can be standardized, CT is now ready for big-time studies of COPD. Standards will need to change as the technology of CT advances. However, the need for new therapeutic initiatives for both $\alpha_{1}$-AT and usual COPD patients dictates that we should proceed with a first effort at standardization as soon as possible.

Since this Editorial was written, the Alpha One Foundation sponsored a workshop on February 2 and 3, 2001 in Miami which gathered together most of the experts in the use of computed tomography for the diagnosis of emphysema. Consensus was reached and their recommendations will be published shortly.

\section{References}

1. Sanders C, Nath PH, Bailey WC. Detection of emphysema with computed tomography: Correlation with pulmonary function tests and chest radiography. Invest Radiol 1988; 23: 262-266.

2. Santis G, Hodson ME, Strickland B. High-resolution computed tomography in adult cystic fibrosis patients with mild lung disease. Clin Radiol 1991; 44: 20-22.

3. Burrows B. An overview of obstructive lung diseases. Med Clin North Am 1981; 65: 455-471.

4. Hayhurst MD, Flenley DC, McLean A, et al. Diagnosis of pulmonary emphysema by computerised tomography. Lancet 1984; 2: 320-322.

5. Gevenois PA, de Maertelaer V, De Vuyst P, Zanen J, Yernault JC. Comparison of computed density and macroscopic morphometry in pulmonary emphysema. Am J Respir Crit Care Med 1995; 152: 653-657.

6. Gevenois PA, De Vuyst P, de Maertelaer V, et al. Comparison of computed density and microscopic morphometry in pulmonary emphysema. Am J Respir Crit Care Med 1996; 154: 187-192.

7. Gevenois PA, Scillia P, de Maertelaer V, Michils A, De Vuyst P, Yernault JC. The effects of age, sex, lung size and hyperinflation on CT lung densitometry. AJR 1996; 167: 1169-1173.

8. Dowson LJ, Guest PJ, Hill SL, Holder RL, Stockley RA. High-resolution computed tomography scanning in $\alpha_{1}$-antitrypsin deficiency: relationship to lung function and health status. Eur Respir J 2001; 17: $1097-$ 1104.

9. Thurlbeck WM. In: Chronic airflow obstruction in lung disease. Philadelphia, W.B. Saunders, 1976.

10. Soejima K, Yamaguchi K, Kohda E, et al. Longitudinal follow-up study of smoking-induced lung density changes by high-resolution computed tomography. Am J Respir Crit Care Med 2000; 161: 1264-1273.

11. Dirksen A, Friis M, Olesen KP, Skovgaard LT, Sorensen K. Progress of emphysema in severe alpha 1-antitrypsin deficiency as assessed by annual CT. Acta Radiologica: 1997: 826-832.

12. Dirksen A, Dijkman JH, Madsen F, et al. A randomized clinical trial of alpha 1-antitrypsin augmentation therapy. Am J Respir Crit Care Med 1999; 160: $1468-1472$.

13. Coxson HO, Rogers RM, Whittall KP, et al. A quantification of the lung surface area in emphysema using computed tomography. Am J Respir Crit Care Med 1999; 159: 851-856. 